This is the post peer-review accepted manuscript of:

Guerra, A., Guidi, F., Dall'Ara, J., \& Dardari, D. (2018). Occupancy Grid Mapping for Personal Radar Applications. In 2018 IEEE Statistical Signal Processing Workshop (SSP) (pp. 766-770). https://doi.org/10.1109/SSP.2018.8450813

The published version is available online at: https://doi.org/10.1109/SSP.2018.8450813

C 2018 IEEE. Personal use of this material is permitted. Permission from IEEE must be obtained for all other uses, in any current or future media, including reprinting/republishing this material for advertising or promotional purposes, creating new collective works, for resale or redistribution to servers or lists, or reuse of any copyrighted component of this work in other works 


\title{
OCCUPANCY GRID MAPPING FOR PERSONAL RADAR APPLICATIONS
}

\author{
Anna Guerra, Francesco Guidi, Jacopo Dall'Ara, Davide Dardari \\ DEI, University of Bologna, via Venezia 52, I-47521 Cesena (FC), Italy. \\ e-mail: \{anna.guerra3,f.guidi,davide.dardari\}@unibo.it
}

\begin{abstract}
Personal radars have been recently proposed starting from the idea that a mobile device can automatically scan and reconstruct a map of the environment thanks to the adoption of millimeter-wave (mm-wave) massive antenna arrays with high-directivity radiation patterns. Unfortunately, despite the extremely narrow-beam achievable, antennas are far from having a pencil beam and, thus, mapping approaches developed for laser-based systems are not appropriate. In this paper, we propose an ad-hoc occupancy grid method for mapping, where the array radiation characteristics are accounted for into the observation model.
\end{abstract}

Index Terms - Occupancy Grid, Mapping, Massive Array, Millimeter-wave, Radar.

\section{INTRODUCTION}

Next fifth generation (5G) of mobile wireless communication foresees the use of mm-wave technology to boost communication at an unprecedented scale, thanks to the large available bandwidth [1]. In addition, the move-up in the frequency spectrum allows to include a large number of antennas into a small area, thus enabling their integration into portable devices $[2,3]$. In this way, such a technological perspective can be exploited to add new functionalities in addition to communication. For example, the laser-like beamsteering allowed by massive arrays at mm-wave can be used to automatically scan and reconstruct the topology of the surrounding environment. Such an idea, namely personal radar, has been recently proposed in theory and its feasibility assessed by experiments [4-6]. In these works, the performance has been investigated through the adoption of a grid-based mapping approach relying on an extended Kalman-Filter (EKF): the environment has been discretized in a grid of cells whose root-radar cross section (RCS) values constitute the state vector to be estimated starting from the backscattered radar response $[7,8]$. To simplify the analysis, the state of the system has been modeled as a Gaussian random vector whose mean vector and covariance matrix are updated during the mapping

The work has been partially funded by the European Commission, under Horizon 2020 project XCYCLE under grant nr. 635975 and IF-GF Marie Skłodowska-Curie project AirSens under grant nr. 793581. process as soon as new measurements are collected [5,9]. The main limitation of this model is that the Gaussian assumption does not capture the underlaying bimodal nature of the phenomenon, i.e., each cell is empty or occupied. In laser-based mapping systems, occupancy grid (OG) methods are usually considered to model this bi-modality by exploiting the basic assumption that laser beam illuminates only one cell per time [10]. This is not the case in radio-based radars where the shape of the radiation pattern is such to illuminate an area composed of several cells, thus making existing OG methods not appropriate due to the inherent cross-correlation between cells that is not zero [11].

In this paper, we propose an OG-based method whose aim is to infer the status (empty/occupied) of the cells describing the environment. Differently from the state-of-the-art, the radiation pattern of the array (including sidelobe beams) is directly taken into account in the observation model to capture the correlation induced by the simultaneous illumination of multiple cells. By means of the considered method, we investigate the impact of different array sizes and we assess the trade-off between the array design complexity (in terms of number of antennas) and the attainable mapping performance in comparison with classical OG methods.

\section{PERSONAL RADAR SIGNAL MODEL}

We now briefly introduce the personal radar concept [5]. We consider the presence of a user with a portable device, equipped with a mm-wave array, moving in an unknown environment. The user device acts as the "personal radar": it automatically steers the main beam of its antenna towards different directions with the purpose to collect information about the topology of the surrounding environment. For each steering direction $\theta_{b}, b=1, \ldots, N_{\text {steer }}$, the radar emits $N_{\mathrm{p}}$ pulses and collects the backscattered response. The received signal can be expressed as

$$
r\left(t, \theta_{b}\right)=\sum_{n=0}^{N_{\mathrm{p}}-1} x\left(t-n T_{\mathrm{f}}, \theta_{b}\right)+n(t)
$$

with $x\left(t, \theta_{b}\right)$ being the useful signal collected when pointing at direction $\theta_{b}$ and $n(t)$ being the additive white Gaussian noise (AWGN) with two-sided power spectral density $N_{0} / 2$. 
Successively, the received signal is passed through an ideal bandpass filter with center frequency $f_{c}$ to eliminate the outof-band noise, thus giving the filtered signal $y\left(t, \theta_{b}\right)$.

As proposed in [5], energy measurements are computed within a time frame $T_{\mathrm{f}}$ divided into $N_{\text {bins }}=\left\lfloor T_{\mathrm{f}} / T_{\mathrm{ED}}\right\rfloor$ discrete time bins of duration $T_{\mathrm{ED}} \approx 1 / W$, with $W$ being the bandwidth of the transmitted signal. Consequently, for each steering direction and for each time bin, the filtered received signal is accumulated over the number of transmitted pulses, i.e. $N_{\mathrm{p}}$, and, then, the corresponding final energy value is given by

$$
e_{b s}=\sum_{n=0}^{N_{\mathrm{p}}-1} \int_{(s-1) T_{\mathrm{ED}}}^{s T_{\mathrm{ED}}} y^{2}\left(t+n T_{\mathrm{f}}, \theta_{b}\right) d t
$$

with $s=1,2, \ldots, N_{\text {bins }}$ being the temporal bin index. Thus, according to $[5,12]$, for each energy bin, the normalized energy measurement can be well approximated by

$$
\Lambda_{b s}=\frac{2}{N_{0}} e_{b s} \approx \frac{1}{\sigma^{2}} \sum_{n=0}^{N_{\mathrm{p}}-1} \sum_{h=(s-1) N_{\mathrm{d}}}^{s N_{\mathrm{d}}} y_{n h}^{2}\left(\theta_{b}\right)
$$

where $N_{\mathrm{d}}=2 W T_{\mathrm{ED}}, \sigma^{2}=N_{0} W$ is the noise variance, and $y_{n h}\left(\theta_{b}\right)$ are for odd $h$ (even $h$ ) the samples of the real (imaginary) part of the equivalent low-pass of $y\left(t+n T_{\mathrm{f}}, \theta_{b}\right)$ taken at Nyquist rate $W$ in each interval $T_{\mathrm{ED}}$. Due to the considered scheme, $\Lambda_{b s}$ is a non-central Chi-square distributed random variable (RV) with $N=N_{\mathrm{p}} N_{\mathrm{d}}$ degrees of freedom, with the non-centrality parameter given by $\lambda_{b s}=2 \gamma_{b s}$, with $\gamma_{b s} \approx \frac{N_{\mathrm{p}}}{N_{0}} \int_{(s-1) T_{\mathrm{ED}}}^{s T_{\mathrm{ED}}} x^{2}\left(t, \theta_{b}\right) d t$. As demonstrated in [12], for large $N_{\mathrm{p}}$ (typically $\left.>50\right), \Lambda_{b s}$ can be considered Gaussian distributed with $\mathbb{E}\left[\Lambda_{b s}\right]=\lambda_{b s}+N$ and $\operatorname{var}\left(\Lambda_{b s}\right)=$ $2\left(N+2 \lambda_{b s}\right)$. In turns, this implies that the generic element $e_{b s}$ can be described as a Gaussian RV with $\mathbb{E}\left[e_{b s}\right]=$ $\frac{N_{0}}{2}\left(\lambda_{b s}+N\right)$ and $\operatorname{var}\left(e_{b s}\right)=\frac{N_{0}^{2}}{2}\left(N+2 \lambda_{b s}\right)$. Then, it is straightforward to obtain

$$
\begin{aligned}
& \mathbb{E}\left[e_{b s}\right] \approx N_{\mathrm{p}} \int_{(s-1) T_{\mathrm{ED}}}^{s T_{\mathrm{ED}}} x^{2}\left(t, \theta_{b}\right) d t+\sigma^{2} N_{\mathrm{p}} T_{\mathrm{ED}}=E_{x}+E_{n} \\
& \operatorname{var}\left(e_{b s}\right)=\sigma_{b s}^{2}=N_{0}\left(2 E_{x}+E_{n}\right)
\end{aligned}
$$

Note that $E_{x}$ depends on the backscattered response of the environment collected when the radar points towards $\theta_{b}$ but it gathers also the energy contributions coming from all the other spatial directions filtered by the array radiation pattern. According to the considered statistical signal model, in the following we introduce an ad-hoc OG mapping method.

\section{OCCUPANCY GRID MODEL}

\subsection{State Vector}

The objective of the estimation process is to infer a probability map of a regular grid of $N_{\text {cells }}$ cells, where each cell composing the environment is represented by the probability of its occupancy. Specifically, the random vector $\mathbf{m}^{(k)}=$ $\left[m_{1}^{(k)}, \ldots, m_{i}^{(k)}, \ldots, m_{N_{\text {cells }}}^{(k)}\right]^{\mathrm{T}}$ is used to model the knowledge of the map at discrete time instant $k$ as a state-space, whereas $\hat{\mathbf{m}}^{(k)}$ is its estimate. The binary $\mathrm{RV} m_{i}^{(k)}=\{0,1\}$ represents the occupancy of the $i$ th grid cell located at position $\mathbf{q}_{i}=\left[x_{i}, y_{i}\right]^{\mathrm{T}}$.

The goal of the mapping process is to infer the belief of the environment map given the history of measurements, i.e.

$$
\hat{\mathbf{m}}^{(k)}=\underset{\mathbf{m}^{(k)}}{\operatorname{argmax}} b\left(\mathbf{m}^{(k)}\right)=\underset{\mathbf{m}^{(k)}}{\operatorname{argmax}} f\left(\mathbf{m}^{(k)} \mid \mathbf{z}^{(1: k)}\right)
$$

where $b\left(\mathbf{m}^{(k)}\right)$ is the belief of the overall map at the discrete time instant $k$ implicitly defined in (5) by the joint conditional probability density function (pdf) $f(\cdot)$, and $\mathbf{z}^{(1: k)}$ is the set of measurements collected until the discrete time $k$. As it can be seen in (5), the mapping problem is described as a maximum a posteriori estimation problem in a high-dimensional space, and thus its direct computation is prohibitive. In order to reduce the complexity, instead of computing the joint conditional pdf $f\left(\mathbf{m}^{(k)} \mid \mathbf{z}^{(1: k)}\right)$, we operate cell-by-cell as

$$
\begin{aligned}
\hat{m}_{i}^{(k)} & =\underset{m_{i}^{(k)}}{\operatorname{argmax}} b\left(m_{i}^{(k)}\right)=\underset{m_{i}^{(k)}}{\operatorname{argmax}} f\left(m_{i}^{(k)} \mid \mathbf{z}^{(1: k)}\right) \\
& =\underset{m_{i}^{(k)}}{\operatorname{argmax}} \frac{f\left(\mathbf{z}^{(k)} \mid m_{i}^{(k)}\right) b\left(m_{i}^{(k-1)}\right)}{f\left(\mathbf{z}^{(k)} \mid \mathbf{z}^{(k-1)}\right)} .
\end{aligned}
$$

Even though (6) implies the independence between the beliefs of the cells, we take into account the dependence arising by the use of non-laser beam arrays in the observation model, as it will be detailed in the following section.

\subsection{Observation Model}

We now describe the adopted observation model capable of capturing the correlations between cells and, hence, to alleviate the condition of independence in (6). We define the vector containing the measured energy bins at time instant $k$ as

$$
\mathbf{e}^{(k)}=\left[e_{11}^{(k)} \ldots e_{b s}^{(k)} \ldots e_{N_{\text {steer }} N_{\text {bins }}}^{(k)}\right]^{\mathrm{T}} .
$$

The generic element $e_{b s}^{(k)}$ does not depend only on the signal backscattered in the steering direction $\theta_{b}$, but also on all the contributions coming from different spatial directions according to the array radiation pattern.

For further convenience, we denote with

$$
\mathbf{c}_{i}^{(k)}=\left[\begin{array}{cccc}
c_{1}^{(k)} & \ldots c_{l}^{(k)} \ldots c_{L_{i}^{(k)}}^{(k)}
\end{array}\right]^{\mathrm{T}}
$$

the ordered vector containing all the cells, except the $i$ th one, at the same distance $d_{i}^{(k)}=\left\|\mathbf{q}^{(k)}-\mathbf{q}_{i}\right\|_{2}$ from the radar position $\mathbf{q}^{(k)}$, i.e. $c_{l}^{(k)}=\left[m_{l}^{(k)}: d_{l}^{(k)}=d_{i}^{(k)}, l \neq i\right] \cdot{ }^{1}$ In other

\footnotetext{
${ }^{1}$ The coordinates in $\mathbf{q}_{i}$ are taken at the center of each cell.
} 


$$
\begin{aligned}
& h_{b s}^{(k)}\left(m_{i}^{(k)}, \mathbf{p}_{j}^{(k)}\right)= \begin{cases}E_{n}+\int_{W} \frac{L_{0}(f)}{\left(d_{i}^{(k)}\right)^{4}}\left(\rho_{i} G^{2}\left(\theta_{i}-\theta_{b}, f\right)+\sum_{l=1}^{L_{i}^{(k)}} g_{j}^{(k)}\left(\rho_{l}\right) G^{2}\left(\theta_{l}-\theta_{b}, f\right)\right) d f & \text { if } s=\left\lfloor\frac{2 d_{i}^{(k)}}{c T_{\mathrm{ED}}}\right\rfloor \\
E_{n} & \text { otherwise. }\end{cases} \\
& h_{b s}^{(k)}\left(\bar{m}_{i}^{(k)}, \mathbf{p}_{j}^{(k)}\right)= \begin{cases}E_{n}+\int_{W} \frac{L_{0}(f)}{\left(d_{i}^{(k)}\right)^{4}}\left(\sum_{l=1}^{L_{i}^{(k)}} g_{j}^{(k)}\left(\rho_{l}\right) G^{2}\left(\theta_{l}-\theta_{b}, f\right)\right) d f & \text { if } s=\left\lfloor\frac{2 d_{i}^{(k)}}{c T_{\mathrm{ED}}}\right\rfloor \\
E_{n} & \text { otherwise. }\end{cases}
\end{aligned}
$$

words, $\mathbf{c}_{i}^{(k)}$ contains all the cells with the same bin index $s$ of the $i$ th cell and, thus, that contribute to the same $e_{b s}^{(k)}$ in (9) regardless the steering index. Starting from (10), we define $\mathbf{P}_{i}^{(k)}=\left[\mathbf{p}_{1}^{(k)}, \ldots, \mathbf{p}_{j}^{(k)}, \ldots, \mathbf{p}_{M_{i}^{(k)}}^{(k)}\right]$ as the matrix of all possible permutations with $\mathbf{p}_{j}^{(k)}$ representing a generic occupancy realization of $\mathbf{c}_{i}^{(k)}$. Consequently, its cardinality is $M_{i}^{(k)}=2^{L_{i}^{(k)}}$ with $L_{i}^{(k)}$ being the cardinality of $\mathbf{c}_{i}^{(k)}$.

Starting from the statistical model of (4), we construct a Gaussian observation model as $\mathbf{z}^{(k)}=\left[\ldots, z_{b s}^{(k)}, \ldots\right]^{\mathrm{T}}$ where the generic element is given by

$$
z_{b s}^{(k)}=\mathbb{E}\left[e_{b s}^{(k)}\right]+v_{b s}^{(k)}
$$

with $v_{b s}^{(k)} \sim \mathcal{N}\left(0, \sigma_{b s}^{2}\right)$ and $\mathbb{E}\left[e_{b s}^{(k)}\right]$ being a function of all the cells, i.e. $\mathbf{m}^{(k)}$, as expressed in (4). In fact, from a statistical perspective, $\mathbf{z}^{(k)}$ represents the observation model conditioned on the overall map, i.e. $f\left(\mathbf{z}^{(k)} \mid \mathbf{m}^{(k)}\right)$. However, the assumption made in the mono-cell problem in (6) is to consider the observation model conditioned only on the single $i$ th cell, i.e. $f\left(\mathbf{z}^{(k)} \mid m_{i}^{(k)}\right)$. This fact does not allow to capture the dependence between cells. To overcome the issue, we formulate the observation model as

$$
f\left(\mathbf{z}^{(k)} \mid m_{i}^{(k)}\right)=\sum_{j=1}^{M_{i}^{(k)}} f\left(\mathbf{z}^{(k)} \mid m_{i}^{(k)}, \mathbf{p}_{j}^{(k)}\right) f\left(\mathbf{p}_{j}^{(k)}\right)
$$

where $f\left(\mathbf{z}^{(k)} \mid m_{i}^{(k)}, \mathbf{p}_{j}^{(k)}\right)=\mathcal{N}\left(\mathbf{h}^{(k)}\left(m_{i}^{(k)}, \mathbf{p}_{j}^{(k)}\right), \mathbf{R}^{(k)}\right)$ with $\mathbf{R}^{(k)}=\operatorname{diag}\left(\sigma_{11}^{2} \ldots, \sigma_{b s}^{2}, \ldots, \sigma_{N_{\text {steer }} N_{\text {bins }}}^{2}\right)$ being the measurement covariance matrix and $\mathbf{h}^{(k)}=\left[\ldots, h_{b s}^{(k)}, \ldots\right]^{\mathrm{T}}$ being a vector of functions that maps the occupancy of each cell to the corresponding energy measurement. The generic term $h_{b s}^{(k)}\left(m_{i}^{(k)}, \mathbf{p}_{j}^{(k)}\right)$ is expressed in (7) with $L_{0}(f)=\frac{P_{t}(f) T_{f} c^{2}}{f^{2}(4 \pi)^{3}}, P_{t}(f)$ being the power spectral density of the transmitted signal, $c$ the speed of light, $G$ the array gain and

$$
g_{j}^{(k)}\left(\rho_{l}\right)=\left\{\begin{array}{lll}
\rho_{l} & \text { if } & p_{j, l}^{(k)}=m_{l}^{(k)} \\
0 & \text { if } & p_{j, l}^{(k)}=\bar{m}_{l}^{(k)}
\end{array}\right.
$$

with $\rho_{l}$ being the RCS of the $l$ th cell and $p_{j, l}^{(k)}$ being the $l$ th element in the $j$ th realization $\left(\mathbf{p}_{j}^{(k)}\right)$. As it can be noticed in (7), $\mathbb{E}\left[e_{b s}^{(k)}\right]$ depends on the contribution of all the cells in $\mathbf{c}_{i}^{(k)}$ through the function $g_{j}^{(k)}(\cdot)$ which relates the occupancy of the cell with its RCS. In (8), it is reported the generic element of $\mathbf{h}^{(k)}$ when the empty cell condition is tested, i.e. when $\left(\mathbf{z}^{(k)} \mid \bar{m}_{i}^{(k)}, \mathbf{p}_{j}^{(k)}\right)$ is considered with $\bar{m}_{i}^{(k)}=1-m_{i}^{(k)}$. Finally, the prior pdf in (12) is approximated by the belief of the cell at the previous time instant, i.e.

$$
f\left(\mathbf{p}_{j}^{(k)}\right) \approx b\left(\mathbf{p}_{j}^{(k-1)}\right)=\prod_{l=1}^{L_{i}^{(k)}} b\left(p_{j, l}^{(k-1)}\right) .
$$

\subsection{Mapping Algorithm}

Given the observation model accounting for the effect of the cell dependence, the belief in (6) becomes

$$
\begin{aligned}
& b\left(m_{i}^{(k)}\right)= \\
& =\frac{\sum_{j=1}^{M_{i}^{(k)}} f\left(\mathbf{z}^{(k)} \mid m_{i}^{(k)}, \mathbf{p}_{j}^{(k)}\right) b\left(\mathbf{p}_{j}^{(k-1)}\right) b\left(m_{i}^{(k-1)}\right)}{f\left(\mathbf{z}^{(k)} \mid \mathbf{z}^{(k-1)}\right)} .
\end{aligned}
$$

Moreover, we can write

$$
\begin{aligned}
& b\left(\bar{m}_{i}^{(k)}\right)=1-b\left(m_{i}^{(k)}\right)= \\
& =\frac{\sum_{j=1}^{M_{i}^{(k)}} f\left(\mathbf{z}^{(k)} \mid \bar{m}_{i}^{(k)}, \mathbf{p}_{j}^{(k)}\right) b\left(\mathbf{p}_{j}^{(k-1)}\right) b\left(\bar{m}_{i}^{(k-1)}\right)}{f\left(\mathbf{z}^{(k)} \mid \mathbf{z}^{(k-1)}\right)} .
\end{aligned}
$$

Taking the ratio between (15)-(16) and after some basic computations, the proposed OG method results in

$$
\begin{aligned}
& b\left(m_{i}^{(k)}\right)= \\
& {\left[1+\frac{\sum_{j} f\left(\mathbf{z}^{(k)} \mid \bar{m}_{i}^{(k)}, \mathbf{p}_{j}^{(k)}\right) b\left(\mathbf{p}_{j}^{(k-1)}\right) b\left(m_{i}^{(k-1)}\right)}{\sum_{j} f\left(\mathbf{z}^{(k)} \mid m_{i}^{(k)}, \mathbf{p}_{j}^{(k)}\right) b\left(\mathbf{p}_{j}^{(k-1)}\right) b\left(m_{i}^{(k-1)}\right)}\right]^{-1} .}
\end{aligned}
$$

Thus, the belief has been expressed not only as a function of the cell considered by the radar, but also of all the others 

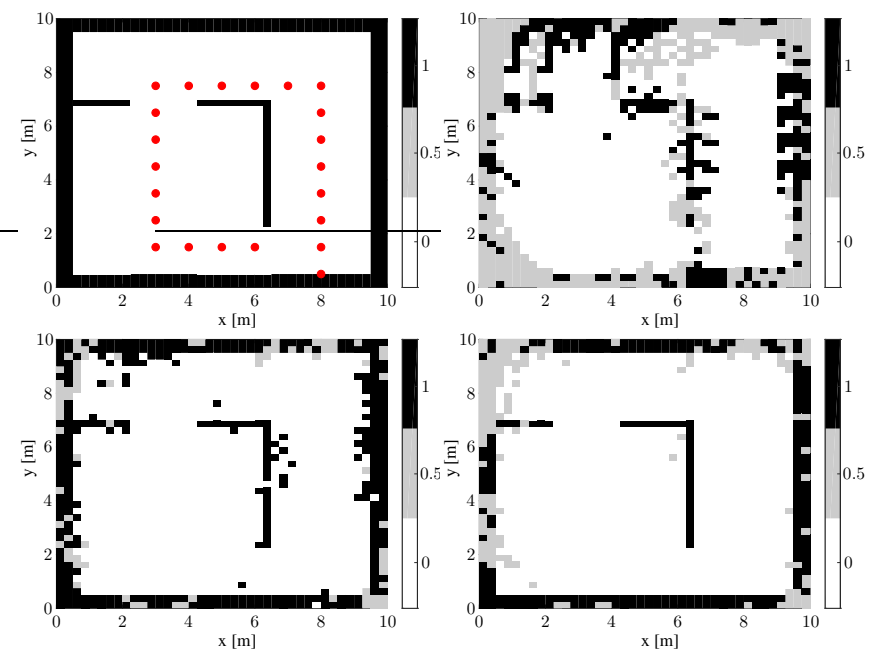

Fig. 1. From the top-left to the bottom-right: (1) Reference map; (2) Map estimated with classical OG and $N_{\mathrm{a}}=16$; (3) Map estimated with the proposed OG and $N_{\mathrm{a}}=16$; (4) Map estimated with the proposed OG and $N_{\mathrm{a}}=100$.

intercepted and contributing to the same set of measurements. In this way, we account for the fact that the array pattern is far from being laser-like in reality.

\section{NUMERICAL RESULTS}

The case study proposed here accounts for an indoor room with size $10 \times 10 \mathrm{~m}^{2}$, discretized in cells of area $0.25 \times$ $0.25 \mathrm{~m}^{2}$. In Fig.1-top left, the considered environment is represented together with the trajectory followed by the personal radar (red dots). Measurements are taken with a step of $1 \mathrm{~m}$ and the steering range spans from $-90^{\circ}$ to $90^{\circ}$ with respect to the heading direction. The color of the map represents the occupancy of each cell, i.e. a cell is marked as either free $\left(b\left(m_{i}\right)=0\right.$, white pixels), occupied $\left(b\left(m_{i}\right)=1\right.$, black pixels) or unknown $\left(b\left(m_{i}\right)=0.5\right.$, grey pixels $)$. The time frame is $T_{\mathrm{f}}=30 \mathrm{~ns}$, whereas the scan time $T_{\text {scan }}=T_{\mathrm{f}} N_{\mathrm{p}} N_{\text {steer }}$ is fixed to $80 \mu \mathrm{s}$ so that the actual number of transmitted pulses $N_{\mathrm{p}}$ changes accordingly. If not otherwise indicated, $N_{\text {steer }}$ is set in accordance to the array beam solid area (i.e., $N_{\text {steer }}=8-12-16-20$ for $\left.N_{\mathrm{a}}=16-36-64-100\right)$ [5]. In addition, we consider $f_{\mathrm{c}}=60 \mathrm{GHz}, W=1 \mathrm{GHz}$ (i.e., $T_{\mathrm{ED}}=1 \mathrm{~ns}$ ), the transmitted power $P_{t}=1 \mathrm{~mW}$ and the noise figure $F=4 \mathrm{~dB}$. The RCS of the walls is $0.01 \mathrm{~m}^{2}$ [5].

Fig. 1 shows three examples of reconstructed OG maps where the color of each pixel is related to the estimated occupancy value. Fig.1-top right and Fig.1-bottom left are obtained for $N_{\mathrm{a}}=16$ and using a classical and the proposed OG with inter-cell dependence, respectively. As it clearly emerges, in the classical OG map, the number of pixels whose occupancy is unknown is higher with respect to the case in which the cell-correlation is considered. The reconstruction

\begin{tabular}{|r|c|c|c|c|}
\hline & \multicolumn{2}{|c|}{ Classical OG } & \multicolumn{2}{c|}{ Proposed OG } \\
\hline$N_{\mathrm{a}}$ & $\Psi$ & $\%_{c=0.5}$ & $\Psi$ & $\%_{c=0.5}$ \\
\hline $4 \times 4$ & 0.23 & 24.75 & 0.07 & 5.53 \\
\hline $6 \times 6$ & 0.031 & 35.81 & 0.01 & 10.53 \\
\hline $8 \times 8$ & 0.023 & 36.11 & 0.001 & 11.30 \\
\hline $10 \times 10$ & 0.0034 & 36.29 & 0 & 10.29 \\
\hline
\end{tabular}

Table 1. IS indexes and percentage of pixels with unknown occupancy value.

fidelity is higher as well. Notably, the quality of the map reconstruction significantly improves when $N_{\text {a }}$ increases, as evident when comparing the two bottom maps in Fig.1.

To obtain a quantitative evaluation of the performance, we now consider a modified version of the image similarity (IS) index in [13], here instead defined as:

$$
\Psi\left(\hat{\mathbf{m}}, \mathbf{m}_{\mathrm{ref}}\right)=\sum_{c \in \mathcal{C}} d\left(\hat{\mathbf{m}}, \mathbf{m}_{\mathrm{ref}}, c\right)
$$

where $\hat{\mathbf{m}}$ is the estimated map, $\mathbf{m}_{\text {ref }}$ the reference map, $\mathcal{C}=$ $\{0,1\}$ the set of occupancy values and

$$
d\left(\hat{\mathbf{m}}, \mathbf{m}_{\mathrm{ref}}, c\right)=\frac{\sum_{q \in \mathbf{q}_{c}} \min \left(d_{M}\left(q, \mathbf{q}_{\mathrm{ref}}\right)=c\right)}{N_{c}}
$$

with $\mathbf{q}_{c}=\left[\mathbf{q}_{i}: \hat{\mathbf{m}}\left[\mathbf{q}_{i}\right]=c, i=1, \ldots, N_{\text {cells }}\right]$ being the vector containing the coordinates of the pixels $\mathbf{q}_{i}$ in the estimated map with occupancy value $c, \mathbf{q}_{\text {ref }}=\left[\mathbf{q}_{i}: \mathbf{m}_{\text {ref }}\left[\mathbf{q}_{i}\right]=c\right]$ the vector of coordinates of the pixels with occupancy value $c$ in the reference map, $d_{M}(\cdot)$ the Manhattan distance and $N_{c}=$ $\left|\mathbf{q}_{c}\right|$ the cardinality of $\mathbf{q}_{c}$. In addition, (18) discards all the contributions coming from the pixels with unknown occupancy information. $^{2}$

Table 1 compares the mapping results using the defined IS score for the classical and the proposed OG approach as well as for different values of $N_{\mathrm{a}}$. Moreover, the percentage of pixels with unknown occupancy $\left(\%_{c=0.5}\right)$ status is reported for both cases. According to this quantitative analysis, we can conclude that the inclusion of the cell-correlations improves the map reconstruction leading to a greater level of similarity with the benchmark map. The same considerations are valid when the number of antennas is increased.

\section{CONCLUSIONS}

In this paper we investigated an ad-hoc OG model tailored for the considered personal radar application. The observation model has been properly designed to include the array radiation pattern. Results show the feasibility of the proposed approach at mm-wave compared to a classical OG method and indicate that accurate mapping performance is attainable even with arrays not extremely massive (i.e. 16 antennas).

\footnotetext{
${ }^{2}$ Numerical solutions can be adopted to reduce the number of pixels with unknown occupancy information arising when probabilities are close to 0 [10].
} 


\section{REFERENCES}

[1] F. Boccardi, R. W. Heath, A. Lozano, T. L. Marzetta, and P. Popovski, "Five disruptive technology directions for 5G," IEEE Commun. Mag., vol. 52, no. 2, pp. 74-80, Feb. 2014.

[2] W. Roh, J.-Y. Seol, J. Park, B. Lee, J. Lee, Y. Kim, J. Cho, K. Cheun, and F. Aryanfar, "Millimeter-wave beamforming as an enabling technology for $5 \mathrm{G}$ cellular communications: theoretical feasibility and prototype results," IEEE Commun. Mag., vol. 52, no. 2, pp. 106-113, 2014.

[3] L. Di Palma, A. Clemente, L. Dussopt, R. Sauleau, P. Potier, and P. Pouliguen, "1-bit reconfigurable unitcell for ka-band transmitarrays," IEEE Antennas Wireless Propag. Lett., vol. PP, no. 99, pp. 1-1, 2015.

[4] F. Guidi, A. Guerra, D. Dardari, A. Clemente, and R. D'Errico, "Joint energy detection and massive array design for localization and mapping," IEEE Trans. Wireless Commun., vol. 16, no. 3, pp. 1359-1371, Mar. 2017.

[5] F. Guidi, A. Guerra, and D. Dardari, "Personal mobile radars with millimeter-wave massive arrays for indoor mapping," IEEE Trans. Mobile Comp., vol. 14, no. 99, 2015.

[6] A. Guerra, F. Guidi, D. Dardari, A. Clemente, and R. D'Errico, “A millimeter-wave indoor backscattering channel model for environment mapping," IEEE Trans. Antennas Propag., vol. 65, no. 9, pp. 4935-4940, Sep. 2017.

[7] H. Durrant-Whyte and T. Bailey, "Simultaneous localization and mapping: Part I," IEEE Robot. Autom. Mag., vol. 13, no. 2, pp. 99-110, 2006.

[8] M. G. Dissanayake, P. Newman, S. Clark, H. F. DurrantWhyte, and M. Csorba, "A solution to the simultaneous localization and map building (SLAM) problem," IEEE Trans. Robot. Autom., vol. 17, no. 3, pp. 229-241, 2001.

[9] A. Guerra, F. Guidi, A. Clemente, R. D'Errico, L. Dussopt, and D. Dardari, "Application of transmitarray antennas for indoor mapping at millimeter-waves," in Proc. European Conf. Netw. Commun. (EuCNC), Jun. 2015, pp. 77-81.

[10] S. Thrun et al., "Robotic mapping: A survey," Exploring artificial intelligence in the new millennium, vol. 1, pp. 1-35, 2002.

[11] S. Thrun, "Learning occupancy grids with forward models," in Proc. IEEE/RSJ Int. Conf. Intell. Robots Sys., vol. 3. IEEE, 2001, pp. 1676-1681.
[12] H. Urkowitz, "Energy detection of unknown deterministic signals," Proc. IEEE, vol. 55, no. 4, pp. 523 - 531, april 1967.

[13] A. Birk and S. Carpin, "Merging occupancy grid maps from multiple robots," Proceedings of the IEEE, vol. 94, no. 7, pp. 1384-1397, 2006. 